\title{
PROJETO MEDALHA: PLATAFORMA ELETRÔNICA PARA APLICAÇÃO DE TESTE DE IMPULSÃO VERTICAL
}

Matheus Piazzalunga Neivock (2) (matheus.neivock@ifms.edu.br), Fabrício César de Paula Ravagnani ${ }^{(1,2)}$ (fabricio.ravagnani@ifms.edu.br), Arthur Lima Moreira ${ }^{(3)}$ (arthurlimamoreira3@gmail.com), Maycon Felipe Mota (2) (mayconfelipemotamw3@gmail.com), Angelo César de Lourenço ${ }^{(2)}$ (angelo.lourenco@ifms.edu.br)

\footnotetext{
(1) Universidade Federal de Mato Grosso do Sul (UFMS); Ciências Humanas e Sociais

(2) Instituto Federal de Mato Grosso do Sul (IFMS); Ciências Exatas e da Terra

(3) Universidade Católica Dom Bosco (UCDB); Ciências Exatas e da Terra
}

RESUMO: O Medalha trata-se de um projeto multidisciplinar e interinstitucional de pesquisa e extensão, desenvolvido em parceria entre a Universidade Federal de Mato Grosso do Sul - UFMS com o Instituto Federal de Educação, Ciência e Tecnologia de Mato Grosso do Sul - IFMS, que visa a avaliação e acompanhamento de atletas de alto desempenho ao longo da carreira (testes físicos, análises clínicas, e outros). O objetivo do projeto foi aplicar intervenções tecnológicas para auxiliar o gerenciamento de dados do Medalha. Diante disto, foram desenvolvidos três produtos: 1) site para o projeto Medalha para divulgação e atendimento dos atletas; 2) sistema web de gerenciamento de dados (internet); 3) protótipo de uma plataforma de salto (teste de impulsão vertical). A plataforma de impulsão desenvolvida consiste num circuito eletrônico capaz de calcular a altura do salto baseado na integral do tempo de flutuação do atleta, projetada para amortecer a queda, bem como possibilitar a ativação automática do ensaio do teste. Para facilitar a transmissão dos dados coletados, estuda-se a possibilidade do envio automático dos resultados via web para o prontuário do atleta, de modo que os profissionais do projeto Medalha tenham acesso instantâneo às informações, proporcionando atendimento com maior precisão e confiabilidade.

PALAVRAS-CHAVE: TECNOLOGIA, PROJETO MEDALHA, IMPULSÃO VERTICAL.

\section{MEDAL PROJECT: ELECTRONIC PLATFORM FOR SARGENT JUMP TEST APPLICATION}

ABSTRACT: The Medal is a multidisciplinary and interinstitutional research and extension project, developed in partnership between the Federal University of Mato Grosso do Sul - UFMS and the Federal Institute of Education, Science and Technology - IFMS, aimed at the evaluation and monitoring of high performance athletes throughout their careers (physical tests, clinical analysis, and others). The objective of the project was to apply technological interventions to assist Medal data management. In view of this, three products were develop: 1) website for the Medal project for the dissemination and care of athletes; 2) web data management system (internet); 3) prototype jumping platform (vertical thrust test). The developed thrust platform consists of an electronic circuit capable of calculating the jump height based on the athlete's float time integral, designed to cushion the fall, as well as enabling automatic activation of the test run. To facilitate the transmission of the collected data, the possibility of automatically sending the results via the web to the athlete's medical record is being study, so that the Medal Project professionals have instant access to the information, providing more accurate and reliable service.

KEYWORDS: TECHNOLOGY, MEDAL PROJECT, VERTICAL THRUST. 


\section{$\mathbf{X I X}$ \\ CENEMI}

\section{INTRODUÇÃO}

De acordo com Barth e Schattenberg e Stollmeier (2013), o uso da tecnologia no esporte pode auxiliar desportistas (em melhora de desempenho ou tratamento de lesões), e também possibilita que a comissão técnica mantenha um histórico do rendimento de seus atletas. Katz (2002) enfatiza que, nos últimos anos, o monitoramento do desempenho dos atletas se tornou praticamente instantâneo, mediante softwares que, analisam os dados durante as competições e enviam os resultados tratados ao técnico.

Infelizmente, essa tecnologia ainda possui um custo elevado. Portanto, viu-se a necessidade do desenvolvimento de novas tecnologias para o auxílio no atendimento de atletas, com ênfase nos testes de desempenho. Dentre esses testes, vale ressaltar o teste de impulsão vertical, que segundo Marins e Giannichi (1998) consiste em aferir a altura máxima do salto de um atleta com auxílio de uma marcação graduada em centímetros (fita, régua ou outros). Diante disto, o projeto Medalha visa analisar os fatores determinantes do alto desempenho e longevidade dos atletas do estado de Mato Grosso do Sul, além de proporcionar atendimento gratuito e desenvolver novas tecnologias para o esporte.

\section{MATERIAIS E MÉTODOS}

Com a finalidade de facilitar a organização do projeto da plataforma de impulsão, ele foi dividido em diversas etapas, desenvolvidas separadamente. Por conta disso, cada parte do presente projeto encontra-se em níveis diferentes de desenvolvimento, como demonstram os subitens abaixo.

\subsection{Teste de impulsão vertical}

Primeiramente, foi realizada uma análise durante a execução de um teste de impulsão, para verificar os componentes que seriam necessários para a construção do protótipo da plataforma, conforme Figura 1.

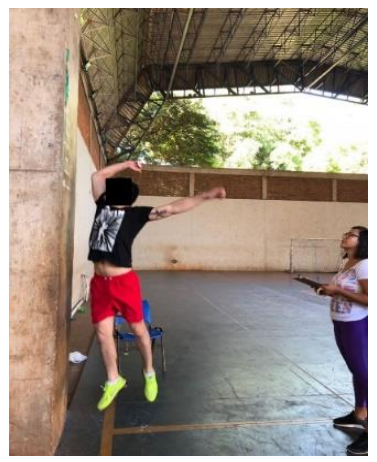

FIGURA 1. Execução do teste de impulsão vertical. Fonte: Autores (2018). 


\section{$\mathbf{X I X}$ \\ CENEMI}

A aplicação do teste consiste nos seguintes passos:

1. O atleta se posiciona ao lado da parede graduada em centímetros, e realiza uma marcação da altura máxima que alcança com os dois pés fixos no chão, levantando um dos braços o mais alto que conseguir, estando rente à parede;

2. Em seguida, o desportista marca a ponta dos dedos da mão mais próxima à parede com pó de giz, para que, ao realizar o salto, possa registrar o ponto mais alto que conseguir;

3. Não são permitidos saltos seguidos de corrida, ou precedidos por outro salto;

4. Durante o teste podem ser realizados dois saltos, com um pequeno intervalo de tempo entre eles.

\subsection{Descrição da fórmula para cálculo da altura}

A partir da observação inicial, verificou-se que o método utilizado é eficaz para análise dos dados de altura, porém, sua precisão pode ser apurada com auxílio da tecnologia, devido a fatores como o tempo de reação do atleta ao marcar o salto, ou a qualidade da marcação. Diante disso, estudou-se a melhor maneira de se descobrir a altura de um salto eletronicamente, adotando então uma adaptação da equação horária da posição em função do tempo, conforme Equação 1.

$$
\Delta S=S_{0}+v_{0} t+\frac{g t^{2}}{2}
$$

A fórmula é constituída de três parcelas, onde:

- So corresponde à posição inicial do atleta, dado em metros;

- $\Delta S$, corresponde à distância percorrida (variação da posição), dada em metros;

- $v_{0}$, representa a velocidade inicial do atleta, dada em metros por segundo;

- $t$, representa o tempo decorrido durante o salto (tempo de flutuação), dado em segundos;

- g, representa a aceleração da gravidade (aproximadamente $9,807 \mathrm{~m} / \mathrm{s}^{2}$ ), dada em metros por segundo ao quadrado;

Felizmente, por se tratar de um sistema onde as condições iniciais (respectivamente, So e vo) são nulas, esta pode ser simplificada, conforme Equação 2. 


\section{XIX \\ CENEMI}

$$
\Delta S=\frac{g^{2}}{2}
$$

Porém, devido à variável desejada ser a altura máxima do salto (dada pela incógnita $\mathrm{h}$, em metros) é preciso fazer uma nova adequação à fórmula, já que a distância percorrida $(\Delta S)$ é equivalente ao dobro da altura alcançada, conforme Equação 3, pois é considerado o trajeto de subida e descida possuem o mesmo comprimento.

$$
\Delta S=2 h \rightarrow 2 h=\frac{g t^{2}}{2}
$$

Após as devidas substituições matemáticas, por apresentar a variação desejadas, possibilitando a obtenção da altura máxima em função do tempo de flutuação do atleta, durante o desenvolvimento do presente trabalho, será utilizada a Equação 4.

$$
h=\frac{g t^{2}}{4}
$$

\subsection{Elaboração do circuito e programação}

Com base na fórmula anteriormente demonstrada, foi possível planejar o programa responsável por sua execução. Optou-se pelo microcontrolador ATmega328 de uma placa arduino UNO, por conta da facilidade de configuração e prototipagem. Sendo assim, utilizou-se inicialmente um sensor resistivo à força, conforme Figuras 2 e 3.

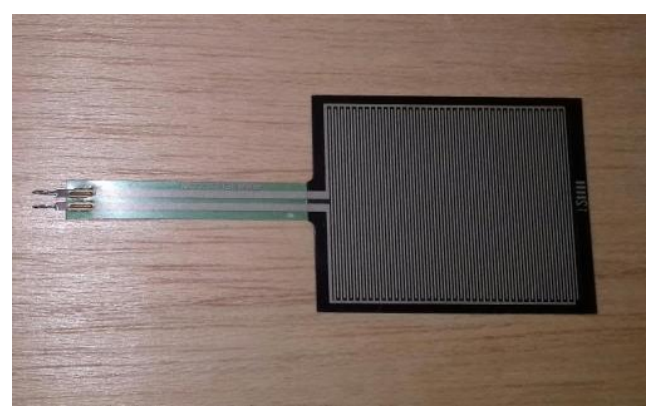

Figura 2. Sensor resistivo à força. Foto: Autores (2018). 


\section{$\mathbf{X I X}$ \\ CIONEMI}

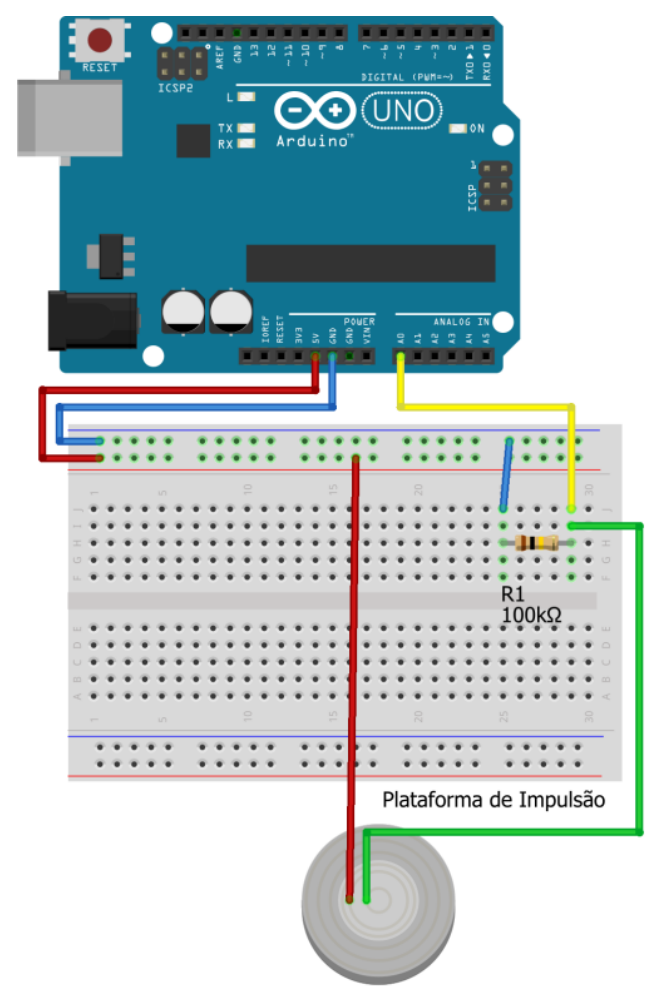

fritzing

Figura 3. Circuito em fritzing de plataforma de impulsão vertical. Foto: Autores (2018).

Em seguida, foi desenvolvido um novo circuito, desta vez utilizando um display LCD 16×2 com módulo 12C, para facilitar as ligações, conforme Figura 4, além servir como monitor serial do cálculo da altura. Para tanto, foi desenvolvido juntamente a programação de um cronômetro eletrônico, para ser possível o cálculo da altura alcançada.

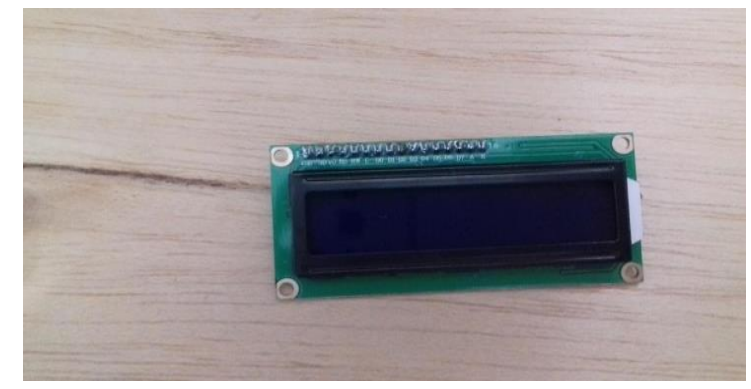

Figura 4. Display LCD 16x2 com módulo I2C. Foto: Autores (2018). 
Como dito anteriormente, o código fonte da plataforma possui um cronômetro. Para tanto, é necessário a utilização de uma biblioteca que possibilite uma função de cronômetro, neste caso a escolhida foi a MsTimer2. Após sua inclusão no código, é feita a configuração do regime de funcionamento, ou seja, são determinadas quais variáveis de tempo serão armazenadas (neste projeto, centésimos e segundos), como mostra a Figura 5.

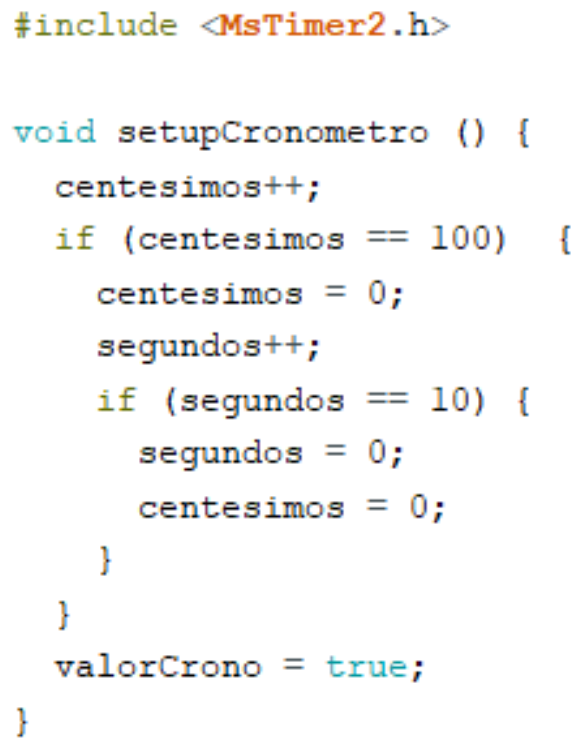

Figura 5. Configuração do cronômetro na IDE arduino. Foto: Autores (2018)

\subsection{Construção da plataforma}

Para a futura alocação do circuito na aplicação do teste de impulsão, foi elaborada uma plataforma constituída por duas placas de MDF, como demonstra a Figura 6, com dimensões de $60 \mathrm{~cm} \times 60 \mathrm{~cm}$, para disponibilizar maior espaço para o posicionamento do atleta durante o teste, conforme Figura 7. 


\section{XIX \\ CONEMI}

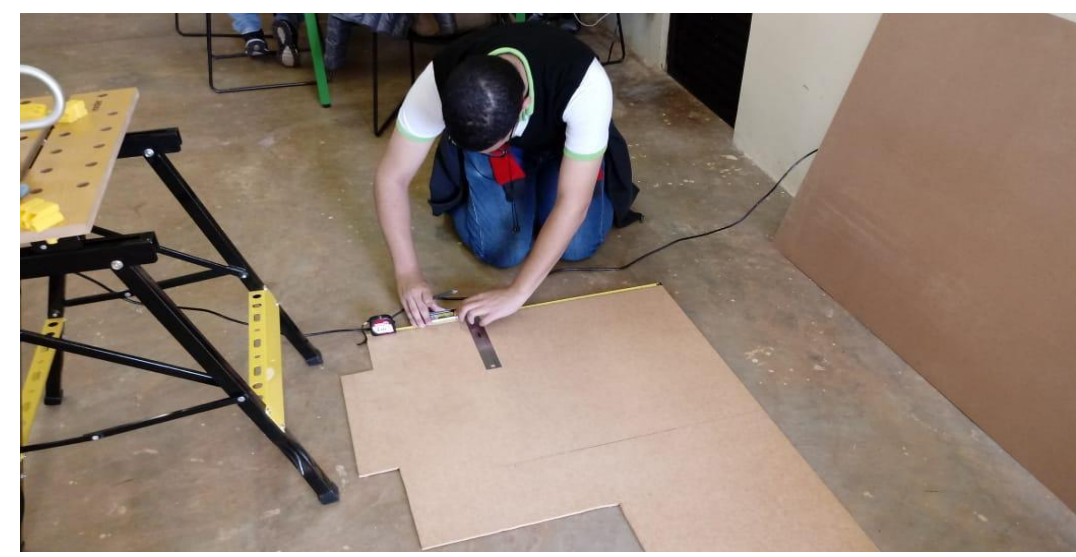

Figura 6. Construção das plataformas de MDF. Foto: Autores (2018).

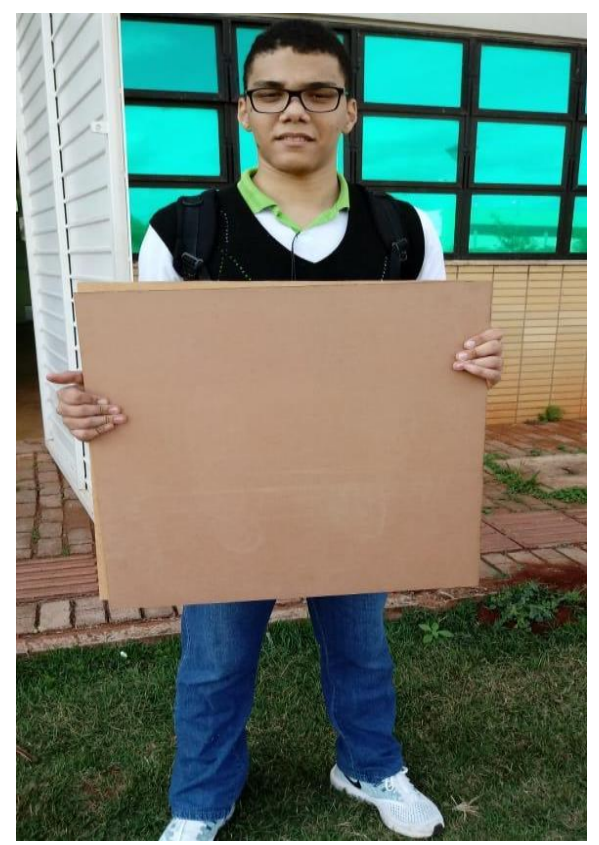

Figura 7. Placas de MDF para construção da plataforma de impulsão. Foto: Autores (2018).

\section{RESULTADOS E CONCLUSÕES}

Atualmente, o presente projeto encontra-se em desenvolvimento. Sendo assim, ainda existem pontos a ser aprimorados em toda sua extensão (hardware e software). Foi construído um protótipo eletrônico da plataforma de impulsão, capaz de realizar o cálculo da altura máxima com base no tempo, como ilustra a Figura 8. 


\section{XIX \\ CONEMI}

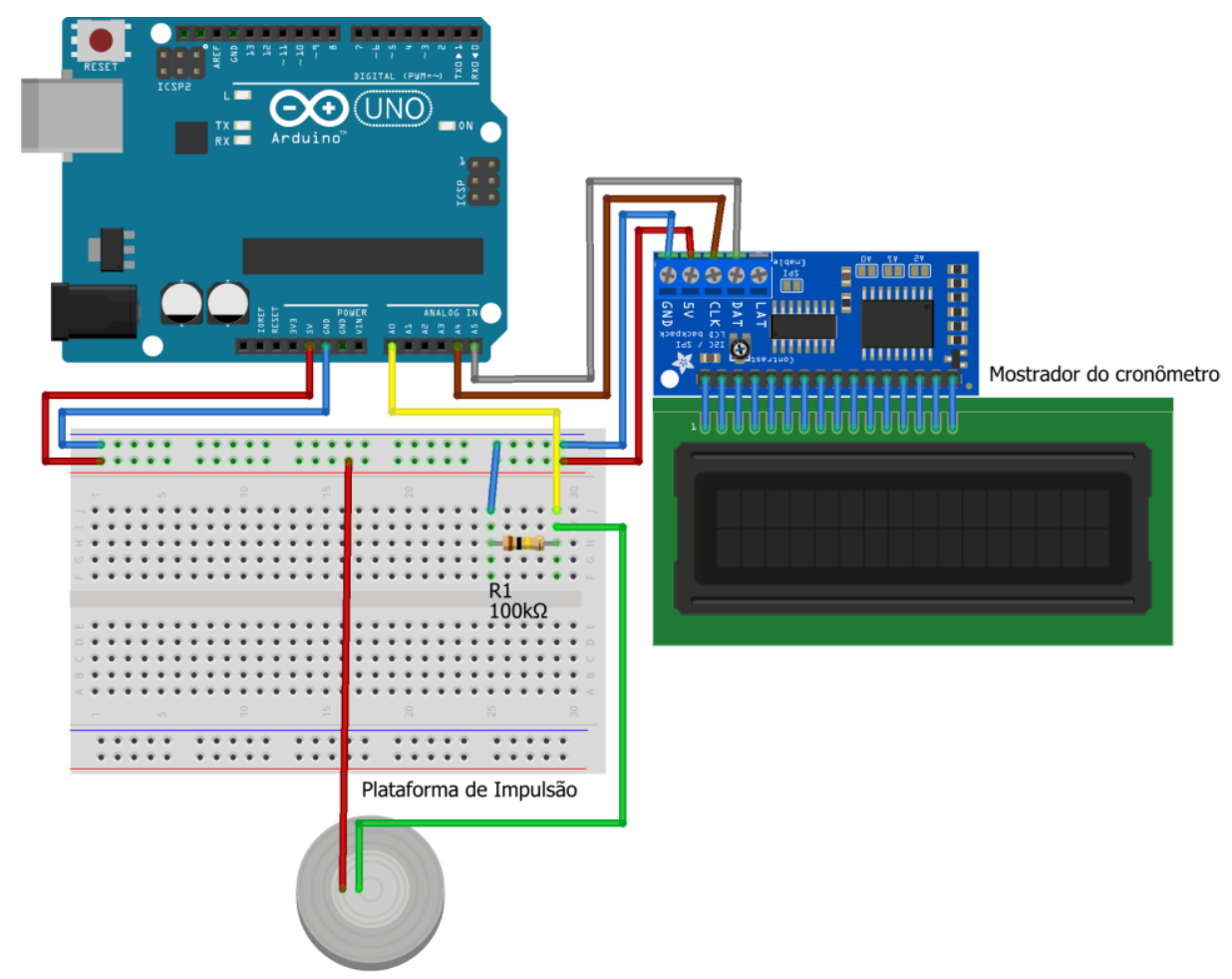

fritzing

Figura 8. Circuito em fritzing da plataforma com cronômetro. Foto: Autores (2018).

\subsection{Problemas encontrados}

Durante o desenvolvimento do projeto foram encontrados diversos problemas, divididos nas seguintes categorias: Software e Materiais (hardware). Os problemas de software, conforme item 3.1.1, correspondem às dificuldades encontradas na programação do circuito, além da incidência de erros lógicos. Já a categoria de materiais, conforme item 3.1.2, representa as dificuldades quanto à qualidade dos sensores e construção do protótipo final.

\subsubsection{Problemas de software}

Embora o software em sua maior parte possua um bom funcionamento, ainda existem problemas a serem estudados e resolvidos para melhor desempenho do programa. Dentre esses, encontra-se o protótipo de comunicação Wi-Fi, de modo que, o circuito envie os resultados ao banco de dados do atleta imediatamente após o teste. Esta intervenção encontra-se em desenvolvimento, utilizando uma placa ESP32 que recebe o resultado por meio de comunicação serial com a placa arduino.

Neste caso, dois problemas foram detectados. O primeiro deles é uma falha de comunicação serial, que impede a leitura correta do resultado obtido no teste pelo sistema do ESP32. Sendo assim, 


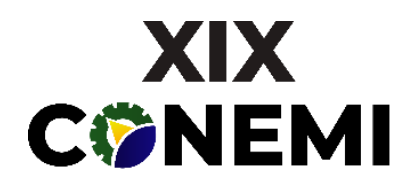

uma das soluções possíveis para este problema, que acarreta em um novo problema, citado posteriormente, é a substituição da placa arduino pela ESP32, tornando-a responsável por todo o gerenciamento do circuito. Porém, para execução desta solução, é necessário o desenvolvimento de um novo código, possivelmente utilizando uma nova biblioteca, para o funcionamento do cronômetro, por conta de conflitos lógicos com o sistema de processamento do ESP32.

\subsubsection{Problemas de materiais}

A partir da prototipagem do circuito com o sensor de força resistivo, constatou-se que, mesmo levando em conta a qualidade de seu sinal, possui ainda um custo elevado por célula. Sendo assim, uma das soluções possíveis é sua substituição por sensores piezoelétricos. Dentre as vantagens da utilização desse tipo de sensor, valem ressaltar a facilidade de manutenção do produto final e por possibilitar a construção de uma plataforma capaz de não apenas detectar pouso, mas também se houve pouso separado de cada um dos pés. Porém, devido ao seu baixo custo, apresentam qualidade de sinal inferior, necessitando tratamento, que pode ser realizado com auxílio de um amplificador operacional. Além disso, por seu polo positivo ser constituído por uma pastilha banhada a ouro, a oxidação pode se mostrar um ponto problemático.

Semelhantemente, é possível que a utilização de madeira como constituinte da estrutura, dificulte a leitura ou acionamento dos sensores. Sendo assim, deve-se realizar, futuramente um estudo mais acurado quanto a influência da estrutura sobre a qualidade do sinal.

\section{REFERÊNCIAS}

BARTH FILHO, J. C.; SCHATTENBERG, L. D.; STOLLMEIER, N. Tecnologias esportivas auxiliando no esporte. Revista Eletrônica do Alto Vale do Itajaí. v.2, n.2, 2013.

KATZ, L. Inovação na tecnologia esportiva: Implicações para o futuro. Revista EF, Rio de Janeiro, v.3, p. 27-32, 2002.

MARINS, João Carlos Bouzas; GIANNICHI, Ronaldo Sergio. Avaliação e prescrição de atividade física: guia prático. Shape, 1998.

MCROBERTS, M. Arduino Básico. Novatec Editora, 2a Edição, Rio de Janeiro, RJ, 512 p. 2015. 